\title{
Single Coronary Anomaly: The Left Main Coronary Artery Originating from the Proximal of Right Coronary Artery
}

\author{
Xinhua $\mathrm{Xu}^{1, \#}$, Enben Guan ${ }^{2, \#}$ and Xuguang Qin*3 \\ ${ }^{1}$ Department of Cardiology, First Affiliated Hospital of Tsinghua University, Beijing, China \\ ${ }^{2}$ Department of Pediatrics, Affiliated Hospital of Qingdao University Qingdao Shandong Province, China \\ ${ }^{3}$ Department of Cardiology, Beijing North Asia Orthopedics Hospital, Beijing, China \\ ${ }^{\#}$ Equally Contributed \\ *Corresponding author: Xuguang Qin, Department of Cardiology, Beijing North Asia orthopedics Hospital, China
}

\begin{abstract}
ARTICLE INFO
Received: 幽 August 27, 2019

Published: 㹃 September 06, 2019

\section{ABSTRACT}

Keywords: Single Coronary Anomaly; Anomalous Left Main Coronary Artery; Coronary Angiography; Computed Tomography
\end{abstract}

Citation: Xinhua Xu, Enben Guan, Xuguang Qin. Single Coronary Anomaly: The Left Main Coronary Artery Originating from the Proximal of Right Coronary Artery. Biomed J Sci \& Tech Res 21(2)2019. BJSTR. MS.ID.003560.
Abbreviations: CAAs: Coronary Artery Anomalies; MSCT: Multi-Slice Computed Tomography; LAD: Left Anterior Descending Coronary Artery; LCX: Left Circumflex Coronary Artery; RCA: Right Coronary Artery; LMCA: Left Main Coronary Artery; PCI: Percutaneous Coronary Intervention; CABG: Coronary Artery Bypass Graft Surgery; SCA: Single Coronary Artery

\section{Introduction}

Coronary artery anomalies (CAAs) are present at birth but are usually asymptomatic and are found during coronary angiography or multi-slice computed tomography (MSCT) detection. Their prevalence is less than $1.3 \%$ based published series [1-3]. The most common coronary anomaly is separate origination of the left anterior descending coronary artery (LAD) and left circumflex coronary artery (LCX) from left sinus of Valsalva. The second most common anomaly is origination of the LCX artery from the right coronary artery (RCA) or right sinus of Valsalva. Herein, we present one case of single coronary artery anomaly that the anomalous left main coronary artery (LMCA) arises from the proximal of the right coronary artery. It is rare case and very important to our clinical practices.

\section{Case Presentation}

A 67-year-old man presented progressive chest pain after physical exertion for ten months, he was admitted to our hospital on November 6th, 2009. He suffered from diabetic mellitus and hypertension for eight years. Coronary angiogram was performed through trans-radial approach. An angiogram demonstrated a single coronary artery: the LMCA originating from the proximal of right coronary artery and then dividing into the left anterior descending artery (LAD) and the left circumflex artery (LCX). In LAO projection there was borderline lesions at the body of the LMCA, it was about $1.5 \mathrm{~cm}$ lesions in length. There was mild narrowing in the mid of LAD segment, and the LCX, RCA were patent (Figures 1-3). As the lesions of LMCA are borderline, neither percutaneous coronary intervention (PCI) nor coronary artery bypass graft surgery (CABG) was performed, the patient was treated by oral medications including aspirin, nitroglycerine, atorvastatin and ACEI drugs. He was free of chest pain during a 24- month follow-up. In order to confirm the origin and course of the anomalous LMCA, a 64-slice computed tomography (MSCT) of the heart was performed on a 64-slice machine (Philips 64 Slice, Philips, Netherland) after 6 months of coronary angiography. The results showed the anomalous left main coronary artery originating from the proximal of the RCA, posterior to the aorta then taking an acute bend to course between the aorta and left atrium (Figures 4\&5). The anomalous LMCA was classified as R-II P subtype. 


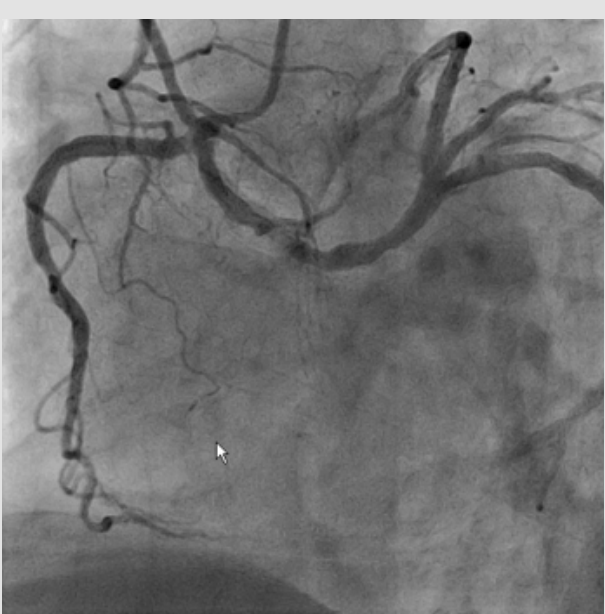

Figure 1: LAO caudal projection shows the anomalous LMCA originating from the proximal of RCA and the lesion of LMCA.

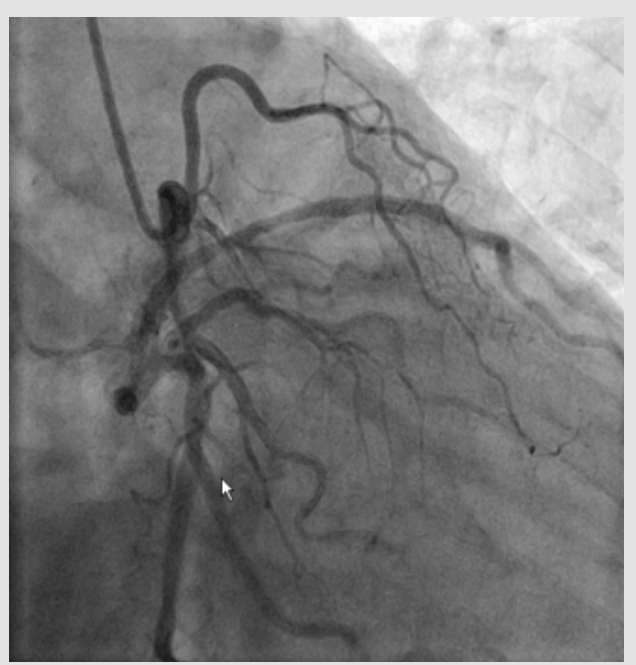

Figure 2: RAO caudal view demonstrates mild narrowing in the mid of LAD segment.

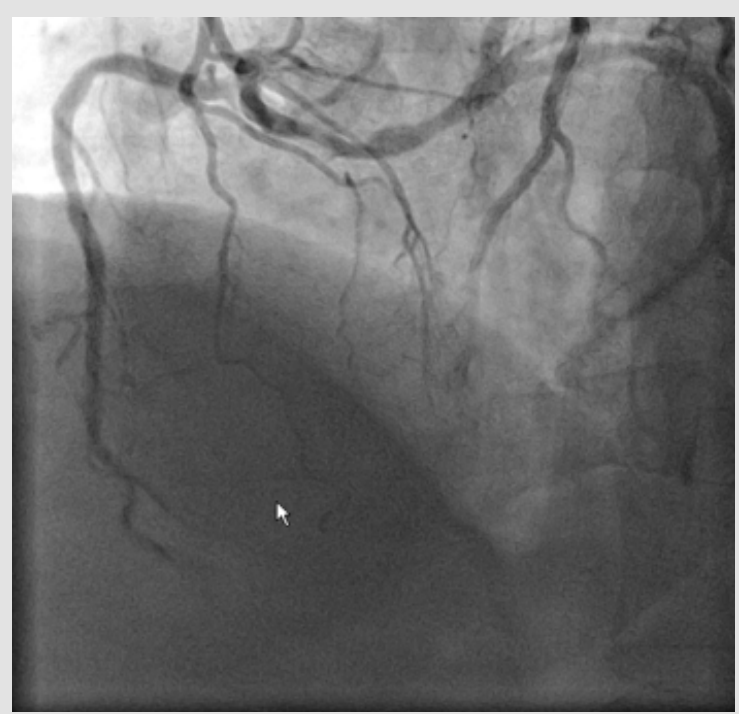

Figure 3: LAO cranial projection demonstrates the borderline long lesion of left main coronary artery.

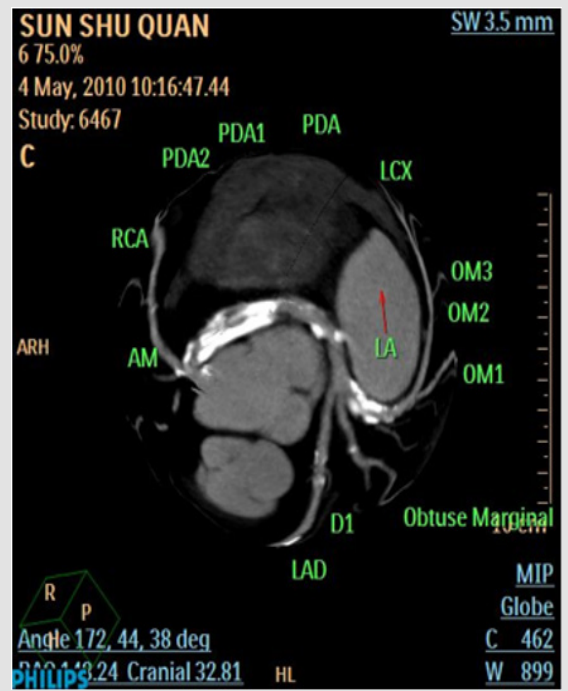

Figure 4: Axial contrast-enhanced multislice computed tomography images. The anomalous LMCA arising from the proximal of RCA.

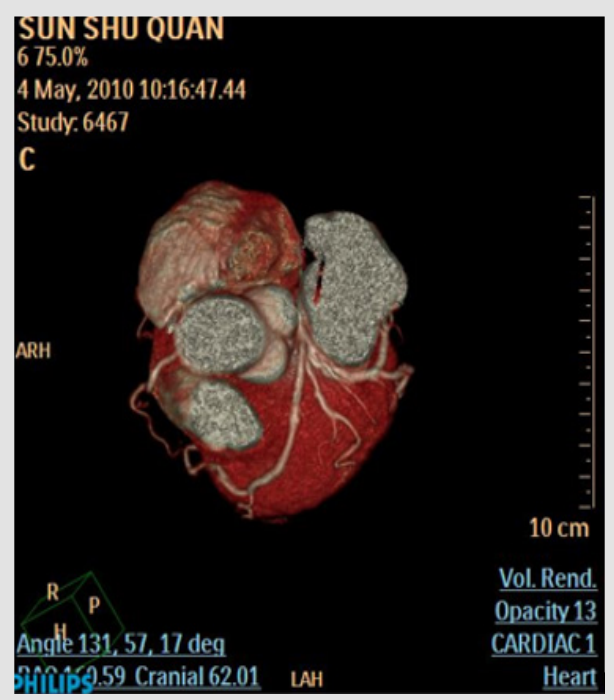

Figure 5: Reconstructed three-dimensional image obtained by volume-rendering technique shows the anomalous LMCA coursed between the aorta and left atrium.

\section{Discussion}

Coronary artery anomalies are present at birth but are usually asymptomatic and are found during coronary angiography or multislice computed tomography (MSCT) detection. Their prevalence is less than $1.3 \%$ based published series [1-4]. The most common coronary anomaly is the left circumflex artery (LCX) arising from the proximal of right coronary artery (RCA). This variant is benign. The second most common coronary anomaly is separate origination of the left anterior descending coronary artery (LAD) and left circumflex coronary artery (LCX) from left sinus of Valsalva. The third most common anomaly is origination of the LCX artery from the right sinus of Valsalva. Single coronary artery (SCA), defined as an artery that arises from an arterial trunk of the coronary sinus of Valsalva and nourishes the entire myocardium, is rare. The incidence of SCA is a rare congenital occurring in approximately 
$0.024 \%$ of the population according to Lipton's reports [2]. It included the following subtype:

a) The anomalous right coronary artery originates from the proximal of left anterior descending coronary artery;

b) The anomalous right coronary artery arises from the distal of left circumflex coronary artery;

c) The anomalous left main coronary artery originates from the proximal of right coronary artery.

The anomalous coronary artery is first designated with " $\mathrm{R}$ " or "L" depending upon whether the ostium is located in the right or left sinus of Valsalva. It is then designated as group I, II, or III anomalies. Group I has anatomical course of either a right or left coronary artery. Group II anomalies arise from the proximal part of the normal right or left coronary artery and cross the base of the heart before assuming the normal position of the inherent coronary artery. Group III describes the anomaly where the LAD and LCX arise separately from the proximal part of the normal RCA. Five anatomical subtypes exist and are classified according to the relationship of the anomalous coronary artery with the aorta and pulmonary artery, i.e., "anterior," "between," "septal," "posterior," and "combined." In this series, the "septal" subtype was the most common, whereas the "between" type was rare [2-5]. However, the left main coronary artery (LMCA) deriving from the proximal of the RCA is extremely rare. Tt's prevalence is less than $0.047 \%$ [1]. Seen in the left ventriculogram, The LMCA may course in front of pulmonary artery (anterior course), through the septum (septal course), between the aorta and pulmonary artery trunk (interarterial course), or behind the aorta (retroaotic course) [6].

The case we presented is the anomalous LMCA arising from the proximal of right coronary artery (RCA). The origin and course of anomalous LMCA was confirmed by MSCT technique [7], the results demonstrated that the anomalous LMCA coursed between the aorta and left atrium. The anomalous LMCA was classified as R-II P subtype (retroaotic course). We bring forth the case in an attempt to highlight their significance. The anomaly is considered to be an independent risk factor for adverse cardiovascular events including sudden cardiac death and dealing with their lesions is a challenge to cardiologists. Coronary anomalies are usually detected during coronary angiography. However, X-ray angiography is limited by its inability to provide information regarding the spatial orientation of the anomalous artery with regarding to the surrounding cardiovascular structures [4,5]. With the help of science and technology, MSCT technique has the potential to accurately visualize the coronary artery [7] and clearly demonstrate the surrounding cardiovascular structures of the anomalous coronary artery [8].

In the present case, the MSCT images clearly demonstrated the origin and course of the anomalous left main coronary artery. It was belonged to R-II P subtype (retroaotic course). So we bring forth the case in an attempt to highlight their significance, and make cardiologist understood what important the anomalies are. A single coronary artery provides a unique challenge to cardiologists when dealing with critical lesions of coronary vasculature, which would otherwise have been easily amenable to angioplasty. Presence of coronary artery anomalies create challenges during coronary angiography, Percutaneous coronary interventions, and coronary artery bypass graft surgery [9]. It is very important to interventional cardiologist and cardiac surgeon in clinical practices [10].

\section{References}

1. Yamanaka O, Hobbs RE (1990) Coronary artery anomalies in 126,595 patients undergoing coronary arteriography. Cathet Cardiovasc Diag 21(1): 28-40.

2. Lipton MJ, Barry WH, Obrez I, Silverman JF, Wexler L, et al. (1979) Isolated single coronary artery: diagnosis, angiographic classification and clinical significance. Radiology 130(1): 39-47.

3. Kardos A, Balsai L, Rudas L, Gaál T, Horváth T, et al. (1997) Epidemiology of congenital coronary artery anomalies: A coronary arteriographic study on a central European population. Cathet Cardiovasc Diagn 42(3): 270-275.

4. Angelini P, Velasco JA, Flamm S (2002) Coronary anomalies incidence, pathophysiology, and clinical relevance. Circulation 105(20): 24492454.

5. Serota H, Barth CW, Seuc CA, Vandirmael M, Aquirre F, et al. (1990) Rapid identification of the course anomalous coronary artery in adults: The "dot and eye" method. Am J Cardiol 65(13): 891-898.

6. Wang A, Pulsipher MW, Jaggers J, Peterson GE, O Laughlin MP, et al. (1997) Simultaneous biplane coronary and pulmonary artery: A novel technique for defining the course of an anomalous left main coronary artery originating from the sinus of Valsava. Cathet Cardiovasc Diagn 42(1): 73-78.

7. Schmid M, Achenbach S, Ropers D, Komatsu S, Ropers U, et al. (2008) Assessment of changes in non-calcified atherosclerotic plaque volume in the left main and left anterior descending coronaries over time by 64-slice computed tomography. Am J Cardiol 101(5): 579-584.

8. Rodenwalt J (2003) Multi-slice computed tomography of the coronary arteries. Eur Radiol 13(4): 748-757.

9. Tariq S, Muddassir S, Patel JK (2012) Single coronary artery anomaly: Branching of left coronary artery from right coronary artery with 2 distinct patterns. J Invas Cardiol 24(4): 67-71.

10. Rudan D, Todorovic N, Starcevic B, Raquz M, Bergovec M, et al. (2010) Percutaneous coronary intervention of an anomalousright coronary artery originating from the left coronary artery. Wien Klin nschr 122(15): 508-510. 


\section{ISSN: 2574-1241}

DOI: 10.26717/BJSTR.2019.21.003560

Xuguang Qin. Biomed J Sci \& Tech Res

(c) (P) This work is licensed under Creative

Submission Link: https://biomedres.us/submit-manuscript.php

$\begin{array}{ll}\text { BIOMEDICAL } & \text { Assets of Publishing with us } \\ \text { RESEARCHES } & \text { - Global archiving of articles } \\ \text { - Immediate, unrestricted online access }\end{array}$

